\title{
So Fun It Hurts - Gamifying an Engineering Course
}

\author{
Gabriel Barata, Sandra Gama, Joaquim Jorge, and Daniel Gonçalves \\ Instituto Superior Técnico / INESC-ID, Rua Alves Redol, 9, 100-029, Lisboa, Portugal \\ gabriel.barata@gist.utl.pt, sandra.gam@gmail.com, \\ jorgej@inesc.pt, daniel.goncalves@inesc-id.pt
}

\begin{abstract}
Good games are good motivators by nature, as they make players feel rewarded and fulfilled, which pushes them forward to persist and resist frustration. Gamification is a novel technique that uses game elements like points and badges, to motivated and engage users into embracing new behaviors, such as improving one's health condition, finances or productivity. In this paper, we present an experiment in which an MSc college course was gamified to improve student interest and engagement. The gamified course led to better learning results and participation. However, there were several negative side effects that detracted from the overall experience. We will describe them, identifying their causes and describe possible alternatives to better tailor the gamified experience, stemming from the analysis of the data gathered so far.
\end{abstract}

Keywords: Education Gamification, Perils, Student engagement, Motivation.

\section{Introduction}

The use of games in non-game contexts is gaining notoriety during the last years. Known as Gamification, it consists in using game elements, instead of full-fledged games, in non-game contexts [1]. It is typically used to keep users engaged and motivated to adopt and perform specific behaviors [2] which makes it of special interest for marketing [3]. Gamification has also been used for a large variety of purposes, like helping people to eat better [4] or to be more productive [5] or eco-friendly [6]

Gamification emerged as a powerful behavior driver, by exploring the motivational power of games and applying it to other domains. Games make players feel rewarded, fulfilled and satisfied, by making them experience what may be called of flow [7], [8]. Flow is what makes players persist and endure, which explains why World of Warcraft players reported to spend 21 hours per week playing the game [9].

Games have been used as motivators with success in education. In different experiments, students from different academic levels were subject to learning with video games, and significant improvements in subject understanding, diligence and motivation were observed [10], [11], [12]. Good games are natural learning machines [13]. Unlike traditional educational materials, games can deliver information on demand and within context, and are balanced so that players do not become either bored or frustrated. This suggests that games and gamification have a great potential to mold human behavior and help people learn new skills, which is also supported by recent 
research. Typical gamified applications rely on game elements such as Points, Badges and Leaderboards as the core of the experience, the so called PBL [19]. While leaderboards allow users to compare themselves with others, points and badges are external rewards for completing certain actions. However, relying solely on these external motivators without considering important human factors like the need to feel competence, autonomy and relatedness [20], will not only fail to engage users, but will also overcrowd any existing interest and internal motivation to perform the behavior in hand [21]. Gamification should be used to boost the user's internal motivation [22].

Jigsaw [14], for example is a gamified application that helps users learn Photoshop, through a jigsaw puzzle that challenges players to match a target image. Although no empirical evaluation was presented, users reported being able to explore the tool and discover new techniques. GamiCAD [15] in turn, is a gamified tutorial system for AutoCAD. By performing line and trimming tasks, users help NASA build a spacecraft to participate in an Apollo mission. Tasks are designed to be challenging and users are encouraged to repeat them until they achieve the required score. When compared to a non-gamified version, results show that users completed tasks faster in GamiCAD and found the experience to be more engaging. Lee Sheldon describes [16] how a conventional learning experience can be designed as a game, without using technology, to engage students and make classes more fun and interesting. Students start with an $\mathrm{F}$ and go all the way up to an A+, by completing quests and challenges, which will reward them with experience points. Khan Academy [17] on the other hand, is a free online service that allows users to learn about several topics, such as algebra, economics or history, by watching videos and then completing exercises. Their progress is rewarded with energy points and badges. Similarly, Codeacademy [18] teaches online students to code in numerous programming languages, also using points and badges to track their progress.

Gamified examples like these suggest a synergic effect between gamification and education. However, little attention has been paid to how these approaches can negatively influence the students' engagement to learn. In this paper we present an experiment in which a college course, Multimedia Content Production (MPC), was gamified, and the problems we found, pointing to possible solutions. We start by describing the course and both the gamified and non-gamified instances, which were deployed in different academic years. Following will be a discussion of the main effects of gamification over student participation and diligence, and we also address in detail the negative side-effects of using a gamified course. We finish by suggesting a few design guidelines for gamified learning experiences.

\section{The MCP Course}

Multimedia Content Production (MCP) is a 5-month long MSc course, in the Information Systems and Computer Engineering degree at Instituto Superior Técnico (University of Lisbon). In the non-gamified year, course evaluation comprised five theoretical quizzes (25\% of total grade), a multimedia presentation (20\%), lab classes (15\%), a final exam (35\%), online participation on the course's forums (5\%) and class 
attendance ( $5 \%$ bonus grade). The final grade was a value between 0 and 20 . In the gamified instance, a new grading system was introduced, where students participated in a game-like experience and were awarded experience points (XP). The evaluation consisted of quizzes (10\%), a multimedia presentation (20\%), lab classes (15\%), a final exam (35\%) and a set of collectible achievements (20\%, plus a 5\% grade bonus). Most achievements were multi-level, for a total of 75 badges that could be won (as well as the corresponding XPs). Compared to the first year, the evaluation method was similar, with achievements replacing online participation and attendance.

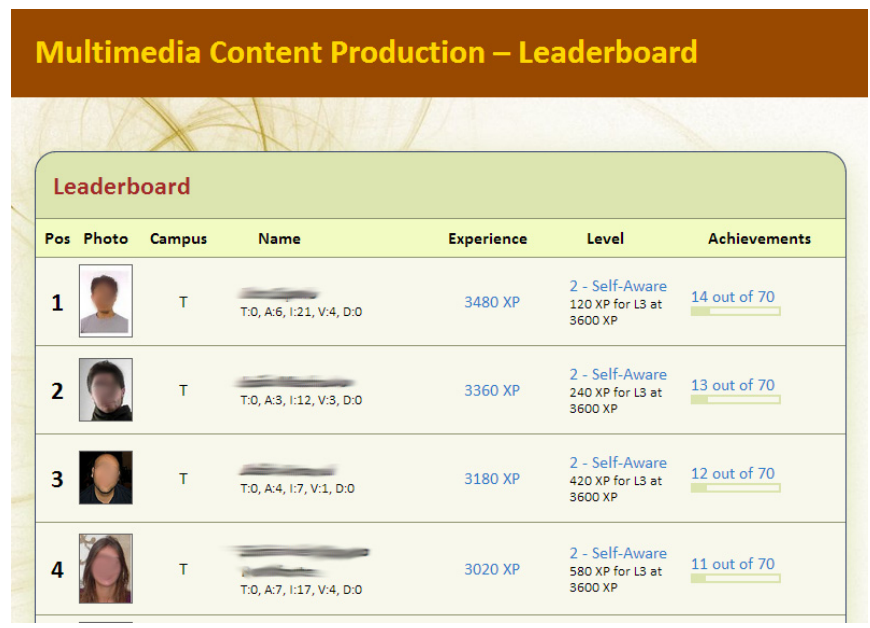

Fig. 1. The MCP Leaderboard

The MCP course was gamified using 6 core game elements: XP, levels, leaderboards, challenges, badges and a skill tree. The leaderboard was the entry point to the whole gamified experience (see Fig. 1). It allowed users to track their progress, explore their own and others' achievement history, and to compare themselves with other classmates. XP and levels served the main purpose of transmitting direct feedback and progress. Students were awarded with XP as they completed course tasks. Every $1200 \mathrm{XP}$ corresponded to a new progress level, in a 20-level scale, which reflected the student's current grade. For example, a student with 12000 XP (10 × 1200 ) would be at level 10 , which corresponds to 10 grade points.

We are aware that some of our game elements do match the PBL formula and work as external rewards. However, we tried to align the goals of the gamified experience with those of the students, which should motivate them by identification and integrated regulation. As posed by the self-determination theory (Deci and Ryan, 2004), these are the most autonomous forms of extrinsic motivation and share some features with the intrinsic forms. We tried to improve three innate needs of intrinsic motivation: competence, by providing positive feedback and displaying progress with points, levels and badges; autonomy, by offering different options of what challenges to pursue and level up; and relatedness, by allowing students feel part of a community and 
participate in the forums. We tried to further improve autonomy with the skill tree (where different paths could be followed, and relatedness, by adding challenges to encourage students to cooperate.

\section{$3 \quad$ Playing the MCP Game}

Overall, the students did well. From a total of 52 students, six reached level 20 (the maximum possible grade!), with no student below level 14, except for an exchange student, a late arrival that was unable to adapt to the course and school (reached level 9, thus failing the course) and a student that gave up at the middle of the semester. These two students will be excluded from the subsequent analysis. Figure 2 summarizes the experience levels reached by the students, and shows the grades to have improved thanks to gamification, when compared to the non-gamified version of the course.

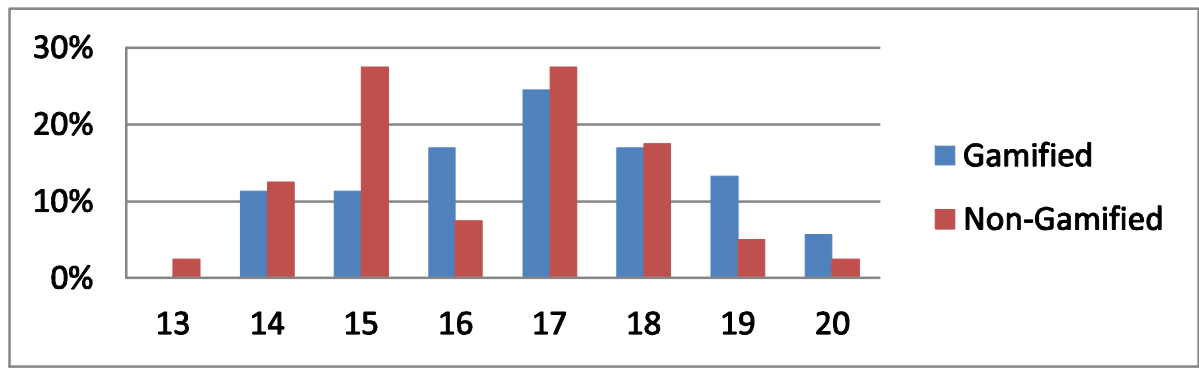

Fig. 2. Percentage of students per final grade

More important than grades, gamification led the students to participate more and be more active learners. Throughout the semester, a total of 2235 posts were made by students, for an average of 139 per week while classes lasted. This contrasts with a much lower figure for the un-gamified version, where only 211 posts overall were made by students. As posts were done mostly to gain certain achievements, for which some work was required, this also means that students worked more often on tasks that exercised the skills learned in the course, with a consequent increase in reinforcement learning, made evident in the final grades.

There were, however, big asymmetries between students. Indeed, the relatively high grades were reached in many different ways, sometimes, as we will see, reluctanty! By carefully studying the ways in which different students played the game throughout the semester, we were able to identify the following typical profiles.

- The Achiever. Achievers (11 students, 21\%) constantly fought for the first place in the leaderboard. Seldom did their position fall below $10^{\text {th }}$ place. These were the students that really enjoyed playing the game, going beyond the minimal requirements just to exercise their skills and have fun. 


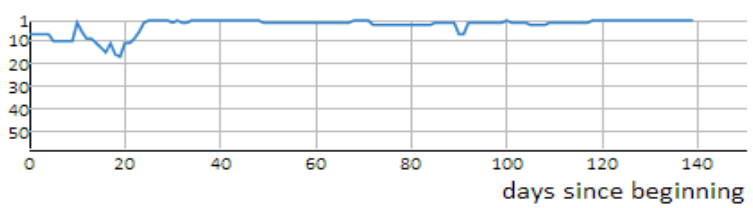

Fig. 3. Typical leaderboard evolution for an Achiever

- The Late Awakener. Late Awakeners (8 students, 15\%) didn't, at first, understand how the course worked. Accustomed to traditional courses with well-defined evaluation moments (a project, an exam, etc.), they neglected the course achievements at first. Once the game progressed and they saw themselves falling behind on the leaderboard, they started participating, often with good results.

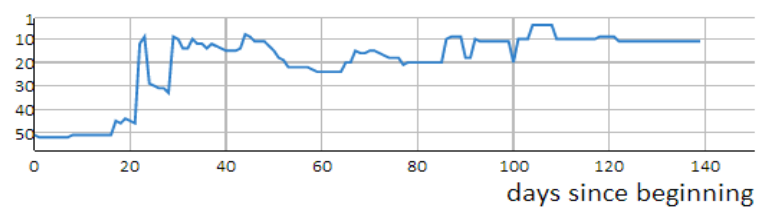

Fig. 4. Typical leaderboard evolution for a Late Awakener

- The Consistent Student. Consistent students remained roughly in the same position throughout the semester, in the middle-bottom part of the leaderboard. There might be some highs and lows, but they clearly spend a consistent (and not very high) effort with the course. This was the most frequently found category, with 21 students (40\%). They typically only went after achievements that were explicitly mentioned in class, with deadlines and, thus, similar to what they know form traditional courses.

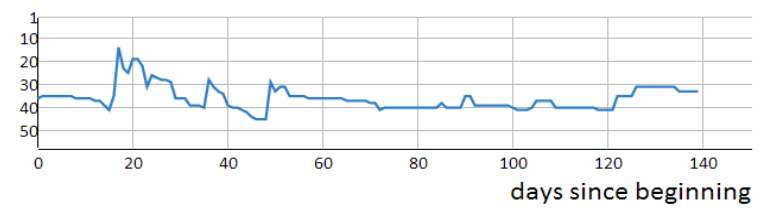

Fig. 5. Typical leaderboard evolution for a Consistent Student

- The Disheartened Student. These (11 students, 21\%) were students that started a strongly at the beginning of the semester but that, after three or four weeks, reverted to a Consistent Student behavior of doing the bare minimum tasks explicitly mentioned by the professors.

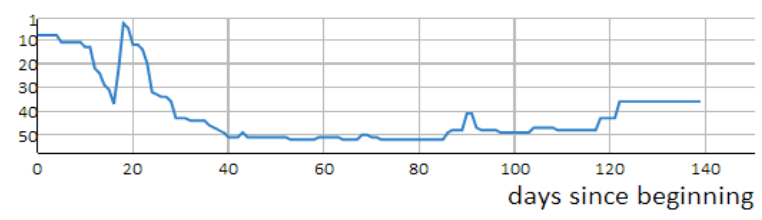

Fig. 6. Typical leaderboard evolution for a Disheartened Student 
In Fig. 7, we can see how students of different types were spread throughout the leaderboard. It is evident that Achievers and Late Awakeners were the best students, while Consistent and Disheartened appear close to the bottom of the list.

\section{- Achiever; - Late Awakener; - Consistent - Disheartened}

Fig. 7. Final leaderboard position of different student types, (from 1, left, to 52, right)

Throughout the course, the differences between these user profiles were made apparent by the nature of comments by the students and the way they participated. Achievers were clearly driving the game forward very actively. Consistent students, while participating, contributed less to the discussion beyond the posts that would strictly earn them achievements. Even so, we can see (Figure 8) that students of all profiles participated. It must be noted that Achievers were atypical in this regard, participating much more than the others. In fact, a set of t-tests shows statistically significant differences only between Achievers and other profiles, but not between the others (with $95 \%$ confidence). This asymmetry led to problems, as we will see below.

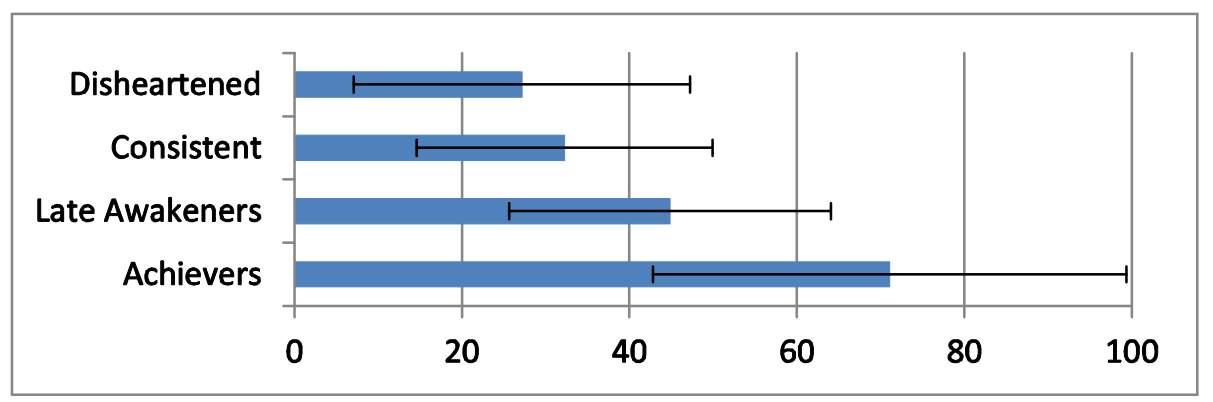

Fig. 8. Average number of posts per student, for the different profiles (error bars: st.dev.)

\subsection{Problems with the Game}

At the end of the game, we asked students to fill in a questionnaire inquiring them on different facets of the game. We had 45 respondents, out of the 52 students. All questions were based on five point Likert scales.

Students were asked whether they had liked the gamified course. Most rated it positively, as seen in Table 1 (avg=3.51, stdev=1.04). Achievers gave it a higher rating (avg=3.89). Late Awakeners, Consistent and Disheartened students gave it ratings of $3.5,3.17$ and 3.67 , respectively. Surprisingly, the students that gave worse ratings to the course were Consistent students. Their ratings ranged from 1 to 5 , with five rating it 1 or 2 , and eight rating it 4 or 5 . This hints at some hidden structure inside this group, not revealed by their leaderboard behavior. Also, it shows that while the Disheartened students appeared to have lost interest in the course, they still liked it more than Consistent students (only one rated it 1 or 2 , and five rated it 4 or 5 ). 
Table 1. Questionnaire responses (on a 5-point Likert scale)

\begin{tabular}{l|lccccc}
\hline & & Total & Achiever & Late-Awakener & Consistent & Disheartened \\
\hline Course Rating & Avg & $\mathbf{3 . 5 1}$ & 3.89 & 3.50 & 3.17 & 3.67 \\
& Stdev & $\mathbf{1 . 0 4}$ & 1.27 & 0.53 & 1.04 & 1.00 \\
\hline Motivation & Avg & $\mathbf{3 . 7 6}$ & 4.22 & 3.50 & 3.56 & 3.78 \\
& Stdev & $\mathbf{1 . 0 5}$ & 0.97 & 0.93 & 1.15 & 0.97 \\
\hline Workload & Avg & $\mathbf{4 . 0 9}$ & 4.89 & 3.88 & 3.89 & 3.89 \\
& Stdev & $\mathbf{0 . 9 2}$ & 0.33 & 0.83 & 1.08 & 0.78 \\
\hline
\end{tabular}

A question about how motivating they found the gamified course yielded a similar pattern. With a total average of $3.76(\mathrm{stdev}=1.05)$, Achievers were by far the most motivated (avg=4.22), followed by Disheartened students (3.78). Future analysis will focus on why students that apparently "gave up" on the course actually liked it more and were more motivated than those that persisted. The questionnaire also had a set of qualitative questions that highlighted the problems discussed below.

Workload. Many users mentioned a high workload as a detrimental factor. When asked to compare the workload in this course to that of others (from 1-much less to 5much more), they replied with a 4.09 average (Table 1). Achievers rated it higher (avg=4.89!), consistently with their struggle for the topmost places in the leaderboard. However, they seldom complained in the qualitative questions. This can mean they were working more for the pleasure of participating and peer recognition. Still, this was an issue for most students. We were convinced that the amount of work hours needed for this course was not dissimilar to the demands of traditional courses (with large programming projects and other tasks throughout the semester). To address this matter, we asked users about it in a post-questionnaire follow up. Responses varied, but a pattern emerged: it is not only the actual workload but the perception of workload that matters. Many courses only require work from students at very limited times throughout the semester (close to a project deadline, an exam, etc.). The gamified course requires them to do much smaller tasks, but requires them continuously. This created the perception that they were "always working for this course", even when the total effort spent was similar to that of other courses.

Comparison Pains. Several students complained about lack of privacy or the visibility of their leaderboard position. They did not want to be compared with, better placed students. Achiever students participated more than could be asked of a typical student. Seeing such a level of activity discouraged others, who felt they could not compete at that level. They resented the fact even while (or, probably, because) getting a better position depended solely on their work. This was exacerbated by the "Talkative" achievement that rewarded classroom participation. Those that didn't participate resented the XP awarded to those that did. Five of the eight students that complained about "Talkative" in the questionnaire were Disheartened students. This reinforces the idea they want to participate, but are intimidated by a level of activity they feel is 
beyond their reach. In traditional courses not all students have the same interest and produce same quality work. This, however, happens silently throughout the semester. The gamified course makes it apparent in real-time.

Reward quality, not quantity. The way the game was set up, students were rewarded for the sole act of participating in the several tasks and challenges posted to them. There were no distinctions in terms of the quality of the work produced. They (rightly) felt it was unfair for contributions of different quality to be rewarded similarly.

Awaking too late. Many of the Consistent students only realized they were getting behind once the course was too far into the semester. As many challenges were timebased, it was now too late for them to fully recover, and many didn't try. Looking at individual achievements, they thought that, since each, individually, isn't worth much, there was no point in working for them. Of course, once their colleagues had amassed sufficient XP points making it apparent the achievement XP add up to a significant amount, they wanted to make up for lost time. By then it was too late. This is where the gaming metaphor breaks down: in a computer game, it is possible to reload and try again. In gamified education (and real-life, in general) that is only possible within very limited boundaries. A subdivision of the Consistent group separating "too late awakeners" is probably relevant and will be considered in future analysis.

Competition vs. Cooperation. Many students complained about the course to be too competitive. However, they did not take advantage of the collaborative features in the game. For instance, an achievement rewarded all students in a lab class if they all did well. It was supposed to serve as an incentive to students helping others. In practice, this never happened. Instead, students with good lab performance complained about groups with lower performance, as it being "their fault" the extra XP hadn't been awarded. This, and similar occurrences, leads us to conclude that, despite the fact they complained about the course being competitive, they are, by nature, competitive, that is, in fact, the culture in our school. Again this was a matter of perception: gamification made explicit that not all students have the same skills (making them resent competition).

\subsection{Design Implications}

From the problems above derives a set of design implications that should be taken into consideration when gamifying this type of course:

- Lighten the pace. The perceived workload must be carefully managed. The intervals between tasks should be carefully chosen to better balance this facet of the game.

- Careful comparisons. Consider other leaderboard types that don't make the direct comparison between students of widely different ratings so easy (displaying only the immediate neighbors, having leaderboards for different "leagues", etc). 
- Reward quality. Estimate the quality of each student's participation and award XPs accordingly. This will increase the amount of work done by the professors but is a requirement for the perceived fairness of the course.

- Make them participate as soon as possible. Many students only want to start playing when it is too late. Tailoring the game experience so that they are compelled to participate (and see meaningful rewards) early on will yield better results.

- Give them the chance to make up for lost time. While some tasks and challenges will always be time-bound, whenever possible it should be allowed for students to address the different challenges in a more unconstrained way.

- Provide means for cooperation. These should not be completely decoupled from competition. Find mechanisms where several students can work together towards a common goal but maintain the ability for students can show off their work.

- Make it all about the game. Several students thought they could neglect the game as some traditional evaluation components (ex: exam) were still in place. Reducing their importance (or getting rid of them altogether) will dispel this illusion.

\section{Conclusions}

Education gamification is a growing trend, with clear advantages in terms of student motivation. However, the gamified experience needs to be carefully tailored not only in absolute terms, but also taking into account the culture and specificities of the students and school. We've shown how problems can arise that detract from the learning process. Most problems mentioned above have to do with the timing for the different game elements and related tasks. These have to be carefully adjusted in order to provide a more balanced gaming experience. Next semester, we will deploy a new version of the gamified course, adjusted based on the lessons learned here. We will explicitely measure engagement and characterize the students trying to fine-tune the profiles defined above. We will also assess the influence of each game element.

Acknowledgements. This work was supported by the Portuguese Foundation for Science and Technology (FCT): individual grant SFRH/BD/72735/2010; project PAELife AAL/0014/2009; and project PEst-OE/EEI/LA0021/2011.

\section{References}

1. Deterding, S., Dixon, D., Khaled, R., Nacke, L.: From game design elements to gamefulness: defining "gamification". In: Proc. 15th International Academic MindTrek Conf. Envisioning Future Media Environments, pp. 9-15. ACM (2011)

2. Shneiderman, B.: Designing for fun: how can we design user interfaces to be more fun? Interactions 11(5), 48-50 (2004)

3. Zichermann, G., Cunningham, C.: Gamification by Design: Implementing Game Mechanics in Web and Mobile Apps. O'Reilly Media, Inc. (2011)

4. http://healthmonth.com/ (February 26, 2013) 
5. Sheth, S., Bell, J., Kaiser, G.: Halo (highly addictive, socially optimized) software engineering. In: Proceeding of the 1st International Workshop on Games and Software Engineering, GAS, vol. 11, pp. 29-32 (2011)

6. Inbar, O., Tractinsky, N., Tsimhoni, O., Seder, T.: Driving the scoreboard: Motivating ecodriving through in-car gaming. In: Proc. CHI 2011 Workshop Gamification: Using Game Design Elements in Non-Game Contexts, ACM (2011)

7. Csikszentmihalyi, M.: Flow: The psychology of optimal experience. Harper Perennial (1991)

8. Nakamura, J., Csikszentmihalyi, M.: Flow theory and research. In: Oxford Handbook of Positive Psychology, pp. 195-206 (2009)

9. Yee, N.: The daedalus gateway (2005), http://www.nickyee.com/daedalus/archives / $001365 . p h p$

10. Coller, B., Shernoff, D.: Video game-based education in mechanical engineering: A look at student engagement. Int. Journal of Engineering Ed. 25(2), 308-317 (2009)

11. Kebritchi, M., Hirumi, A., Bai, H.: The effects of modern math computer games on learners' math achievement and math course motivation in a public high school setting. British Journal of Educational Technology 38(2), 49-259 (2008)

12. Squire, K., Barnett, M., Grant, J.M., Higginbotham, T.: Electromagnetism supercharged!: learning physics with digital simulation games. In: Proc. ICLS 2004, International Society of the Learning Sciences, pp. 513-520 (2004)

13. Gee, J.P.: What video games have to teach us about learning and literacy. Comput. Entertain. 1(1), 20 (2003)

14. Dong, T., Dontcheva, M., Joseph, D., Karahalios, K., Newman, M., Ackerman, M.: Discovery-based games for learning software. In: Proc. CHI 2012, pp. 2083-2086. ACM, New York (2012)

15. Li, W., Grossman, T., Fitzmaurice, G.: Gamicad: a gamified tutorial system for first time autocad users. In: Proc. 25th Annual ACM Symposium on User Interface Software and Technology, UIST 2012, pp. 103-112. ACM, New York (2012)

16. Sheldon, L.: The Multiplayer Classroom: Designing Coursework as a Game. Course Technology PTR (2011)

17. https: / /www. khanacademy . org/ (February 26, 2013)

18. http: / / www. codecademy. com/ (February 26, 2013)

19. Werbach, K., Hunter, D.: For the Win: How Game Thinking Can Revolutionize Your Business. Wharton Digital Press (2012)

20. Deci, E., Ryan, R.: Handbook of self-determination research. University of Rochester Press (2004)

21. Rigby, S., Ryan, R.: Glued to games: How video games draw us in and hold us spellbound. Praeger (2011)

22. Deterding, S.: Gamification: designing for motivation. Interactions 19(4), 14-17 (2012) 